\title{
Chitosan Nano-cocktails Containing Both Ceria and Superparamagnetic Iron Oxide Nanoparticles for Reactive Oxygen Species-Related Theranostics
}

Yuao Wu ${ }^{1,2}$, Run Zhang ${ }^{1}$, Huong D.N. Tran ${ }^{1,2}$, Nyoman D. Kurniawan ${ }^{4}$, Shehzahdi S. Moonshi ${ }^{1}$, Andrew K. Whittaker ${ }^{1,5}$, Hang T. Ta ${ }^{1,2,3^{*}}$

${ }^{1}$ Australian Institute for Bioengineering and Nanotechnology, University of Queensland, St Lucia, Queensland 4072, Australia

${ }^{2}$ Queensland Micro- and Nanotechnology, Griffith University, Nathan, Queensland 4111, Australia

${ }^{3}$ School of Environment and Science, Griffith University, Nathan, Queensland 4111, Australia

${ }^{4}$ Centre of Advanced Imaging, University of Queensland, St Lucia, Queensland 4072, Australia

${ }^{5}$ ARC Centre of Excellence in Convergent Bio-Nano Science and Technology, The University of Queensland, Brisbane, Queensland 4072, Australia

*Correspondence: Hang T. Ta (h.ta@griffith.edu.au) 


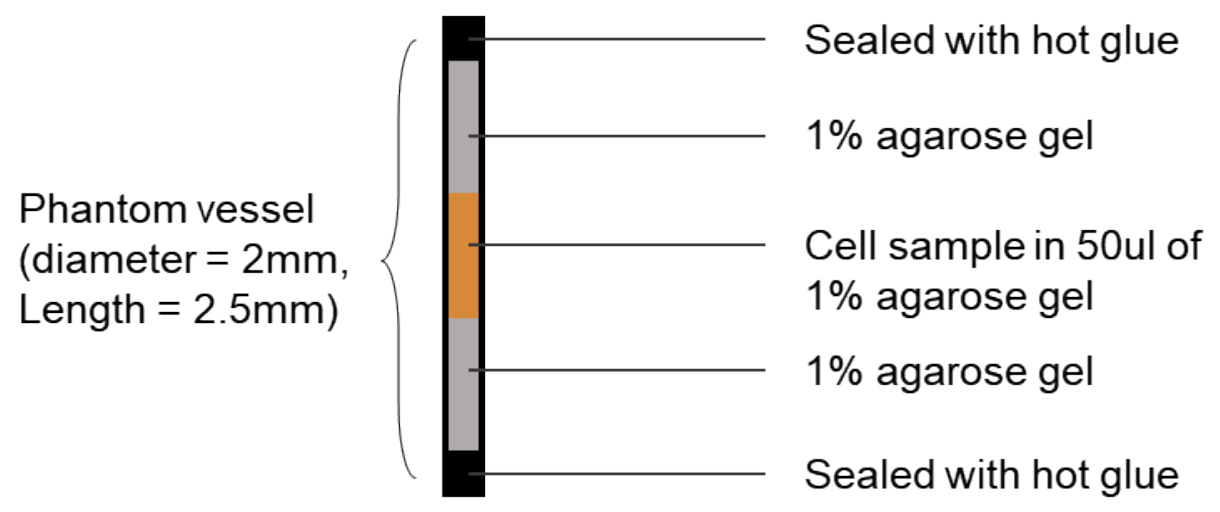

Scheme S1. Schematic Illustration of the Preparation of Phantom Vessel for MRI

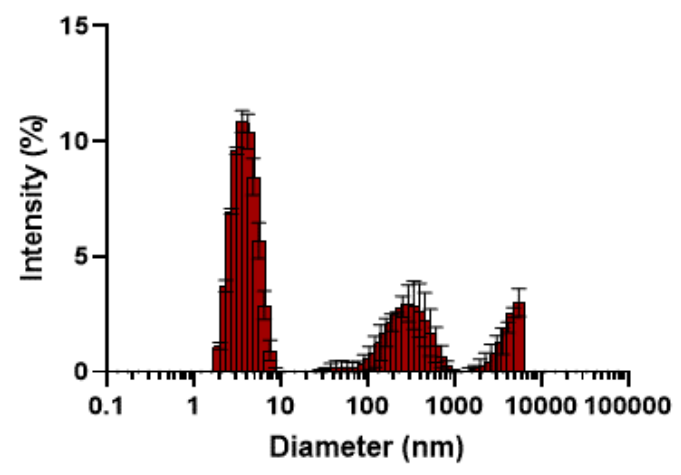

Fig S1. Assessment of the stability of CO-TSC in water for 24 hours. Size of CO-TSC in water solution was measured by DLS. 

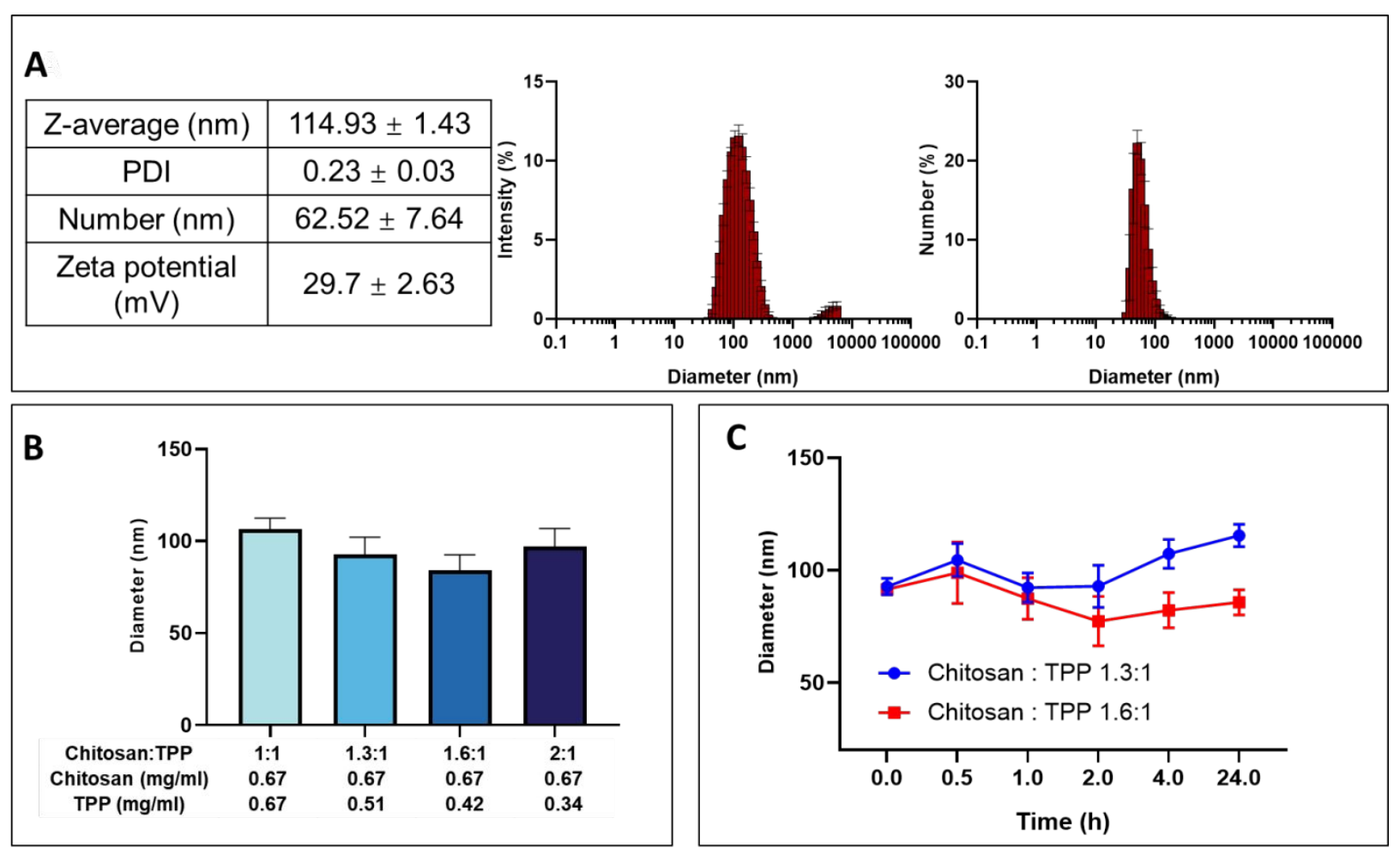

Fig S2. Characteristics of Chit-TPP. (A) Size and zeta potential of Chit-TPP measured by Zetasizer. Zeta potential data showed that Chit-TPP nanoparticle was positively charged. (B) DLS size distribution of the Chit-TPP nanoparticles. (C) Size of the Chit-TPP nanoparticles after synthesis with different concentrations of TPP. Different concentration of TPP in $2.5 \mathrm{ml}$ of $\mathrm{H}_{2} \mathrm{O}$ $(0.67 \mathrm{mg} / \mathrm{ml}, 0.51 \mathrm{mg} / \mathrm{ml}, 0.42 \mathrm{mg} / \mathrm{ml}$ and $0.34 \mathrm{mg} / \mathrm{ml})$ was pumping into $0.1 \%(\mathrm{w} / \mathrm{w})$ of chitosan solution during synthesis. (D) Size of the Chit-TPP nanoparticles of different incubation time. Different concentration of TPP in $2.5 \mathrm{ml}$ of $\mathrm{H}_{2} \mathrm{O}(0.51 \mathrm{mg} / \mathrm{ml}),(0.42 \mathrm{mg} / \mathrm{ml})$ was pumped into $0.1 \%(\mathrm{w} / \mathrm{w})$ of chitosan solution (chitosan:TPP ratios of 1.3:1 and 1.6:1, respectively) and stirred for $0.5 \mathrm{~h}, 1 \mathrm{~h}, 2 \mathrm{~h}, 4 \mathrm{~h}, 24 \mathrm{~h}$. 

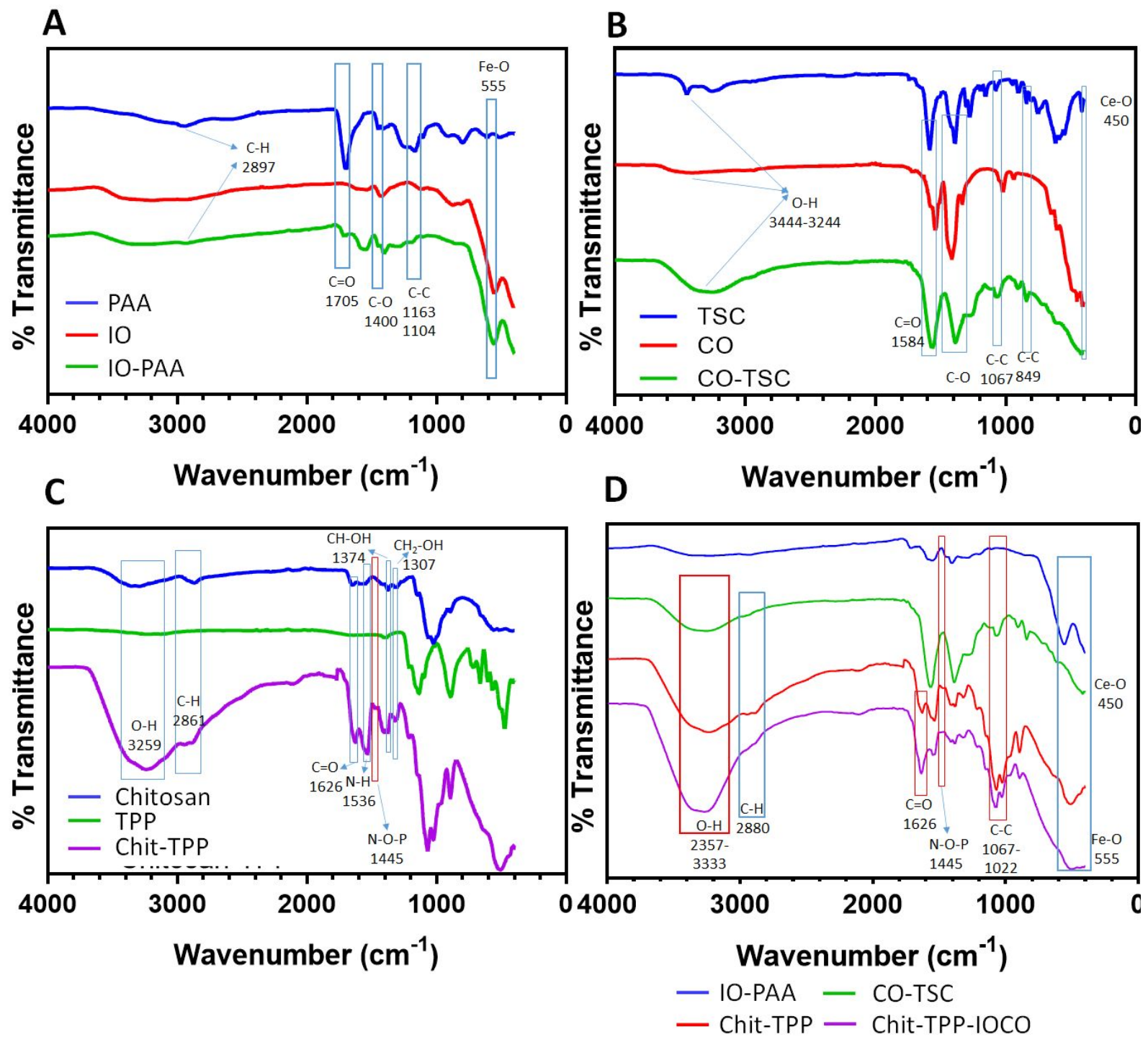

Fig S3. FTIR spectroscopy of nanoparticle and their relevant materials. (A) FTIR spectroscopy of PAA IO and IO-PAA. PAA, IO and IO-PAA powders were dried, followed by FTIR measurement. Peaks at around $2898 \mathrm{~cm}^{-1}$ showed C-H bonds, $1705 \mathrm{~cm}^{-1}$ indicated $\mathrm{C}=\mathrm{O}$ bonds, $1400 \mathrm{~cm}^{-1}$ showed C-O bonds, 1104 to $1163 \mathrm{~cm}^{-1}$ showed C-C bonds and at $555 \mathrm{~cm}^{-1}$ were $\mathrm{Fe}-\mathrm{O}$ bonds. According to the PAA and iron oxide structure and infrared spectroscopy, PAA was successfully coated on the IO-PAA nanoparticles. (B) FTIR spectroscopy of TSC, CO and CO-TSC. (C) FTIR spectroscopy of chitosan, TPP and Chit-TPP nanoparticle. (D) FTIR spectroscopy of IO-PAA, CO-TSC, Chit-TPP and Chit-TPP-IOCO nanoparticles. 